\title{
A geometric framework for phase synchronization in coupled noisy nonlinear systems
}

\author{
J.Balakrishnan* \\ Instituut-Lorentz for Theoretical Physics, Universiteit Leiden, \\ Postbus 9506, 2300 RA Leiden, The Netherlands.
}

P.A.C.S. numbers : 05.45.-a， 05.45.Xt，05.40.-a， 02.90.+p， 89.75.-k

\begin{abstract}
A geometric approach is introduced for understanding the phenomenon of phase synchronization in coupled nonlinear systems in the presence of additive noise. We show that the emergence of cooperative behaviour through a change of stability via a Hopf bifurcation entails the spontaneous appearance of a gauge structure in the system, arising from the evolution of the slow dynamics, but induced by the fast variables. The conditions for the oscillators to be synchronised in phase are obtained. The role of weak noise appears to be to drive the system towards a more synchronized behaviour. Our analysis provides a framework to explain recent experimental observations on noiseinduced phase synchronization in coupled nonlinear systems.
\end{abstract}

*E-mail: janaki@lorentz.leidenuniv.nl, janaki@rri.res.in 


\section{INTRODUCTION}

Synchronization phenomena occur abundantly in nature and in day to day life. A few well known examples are the observations in coupled systems such as pendulum clocks, radio circuits, swarms of light-emitting fireflies, groups of neurons and neuronal ensembles in sensory systems, chemical systems, Josephson junctions, cardiorespiratory interactions, etc. Starting from the observation of pendulum clocks by Huygens, a vast literature already exists which studies synchronization in coupled nonlinear systems — in systems of coupled maps as well as in oscillators and networks [1 \& references therein]. In recent times, different kinds of synchronization have been classified — mutual synchronization, lag synchronization, phase synchronization and complete synchronization [1-3].

Many of these studies aim to understand the properties exhibited by the systems once they synchronize or exhibit phase-locking. A comprehensive understanding still seems to be lacking when one seeks to explain why the systems synchronize. In this paper we introduce a geometric approach in order to address

this fundamental issue. We aim to understand here the reason for the occurrence of synchronized and phase-locked behaviour in coupled nonlinear systems which are subject to weak additive noise.

We consider a system of $n$ nonlinear oscillators which are coupled. We determine the conditions which the dynamically evolving variables of the system must satisfy in order that the various oscillators constituting the full system synchronize in phase. Our study at the moment does not include chaotic systems. We find that the presence of weak noise assists in bringing about phase synchronization.

In section 2, we introduce a geometrical approach to discuss coupled dynamics. We have adapted the methods which Wilczek \& Shapere developed [4-6] to understand self-propulsion of organisms by shape deformations in fluids at low Reynolds number, for discussing the deformations and changes in the orbit structure in phase space as the system evolves in time. In section 3 we discuss the dynamics of coupled oscillatory systems in the presence of additive noise at the close proximity of a Hopf bifurcation. We show the spontaneous emergence of a non-trivial gauge structure for such a system arising from the slow degrees of freedom, and induced by the fast variables, and associate it with the geometric approach introduced in section 2. In section 4, we obtain the condition required to be satisfied by any two oscillators to exhibit phase synchronization \& phase locking. Section 5 summarizes the main results of the paper and points out 
directions for future studies.

Our work is motivated by the need for a theoretical understanding of recent experiments on chemical oscillators [7] and numerical simulations [8] which show noise-induced phase synchronization in coupled nonlinear systems. (See also [9],[29]).

\section{THE GEOMETRY UNDERLYING THE DYNAMICS OF COUPLED SYSTEMS}

In a series of beautiful papers [4-6], Shapere \& Wilczek established a geometric framework to discuss the motion of deformable objects in the absence of applied external force. We adopt these methods to understand a fundamental phenomenon in nonlinear dynamics, namely phase synchronization in coupled dynamical systems.

In the first section, the main idea underlying the paper is developed, which is based on the approach used in [4-6] for a deterministic system. Elaborating along these lines, in the following sections we have studied a general system of coupled nonlinear oscillators under the influence of additive Gaussian white noise and we find the conditions under which the coupled units within the full system can exhibit phase-locked behaviour and phase synchronization.

We consider a system of $n$ coupled generalised oscillators $q(x, t)$ where the state variables $q$ could in general be functions not only of time, but could also depend on a set of additional variables $x$, say spatial variables when there is a metric structure associated with the variables:

$$
\dot{q}_{i}=f_{i}\left(q_{1}, q_{2}, \ldots, q_{n}, \mu_{j}\right), \quad i=1, \ldots, n, \quad j=1, \ldots, p
$$

Thus $q_{i}$ include also extended systems where the individual elements could mutually influence each other through a distance-dependent interaction between the elements. For instance in the case of coupled chemical

oscillators [8], $q_{i}$ would denote concentrations which have spatial dependence. Another example occurs in biological information processing where neurons interact in ensembles.

We study the simplest case in which, in the absence of couplings, each of the $n$ subsystems admits oscillatory solutions for some parameter values $\mu_{j}$. Switching on the mutual coupling between these oscillators results in the emergence of a collective behaviour. It is then appealing to view the collective behaviour as having arisen as a result of some sort of communication between different points in the configuration space. Thus 
one is led to a geometrical description of the system's dynamical evolution.

For simplicity, we restrict ourselves in this paper to the case in which the collective dynamics also exhibits limit cycle behaviour emerging via one or more Hopf bifurcations. The more general situation which includes chaotic orbits for the uncoupled dynamics is not considered here. We define the configuration space of the full system as the space of all possible flow lines and closed paths. We consider the situation when there is no external driving force, so that the space of all possible contours is the space of oriented contours centred at the origin.

In the absence of mutual couplings, the space of contours consists of oriented closed orbits, each orbit inclined at an angle with respect to the other. If we now turn on the mutual couplings between these $n$ subsystems gradually, each of the orbits would gradually get deformed, going through a sequence of shape changes, and resulting subsequently in a net rotation for it. The problem of interest is to link the dynamical variables of the system with the net rotation induced by a change of shape of the orbits for each of the $n$ oscillators in phase space.

The relative orientations of any two contour shapes can be compared by fixing coordinate axes for each. Since there exists a degeneracy in the possible choice of axes one can make at each point in the space of contour shapes, each set of reference frame we can choose from being isomorphic to $E_{n}$, a gauge structure is therefore induced in this space which facilitates going from one particular choice of axes to another.

In [4-6], the problem of self-propulsion at low Reynold's number made possible solely through shape deformations was discussed. Each choice of reference frame fixed to a shape, which assigned a "standard location" in space for each shape, was associated with the motion and location of any arbitrary shape in relation to its standard location. We follow their methods closely to discuss deformations of the oriented contours in the space of contour shapes.

Following $[5,6]$, the sequence of oriented contours $S(t)$ can be similarly related to the sequence of the corresponding chosen reference standard contour shapes $S_{0}(t)$ by a rigid displacement $\mathcal{R}(t)$ :

$$
S(t)=\mathcal{R}(t) S_{0}(t)
$$


where in general, an $n$-dimensional motion $\mathcal{R}$ includes both rotations $R$ and a translation $l$ :

$$
[R, l]=\left(\begin{array}{ll}
R & l \\
0 & 1
\end{array}\right)
$$

where $R(t)$ is an $n \times n$ rotation matrix and stands for a sequence of time-dependent rigid motions. The contour boundaries are parametrized by the control parameters $\mu_{i}$, for each of which the rigid motion $\mathcal{R}$ acts on the vector $\left[S_{0}(\mu), 1\right]^{T}$. The physical contours $S(t)$ are invariant under a local change

$$
\tilde{S}_{0}=\Omega\left[S_{0}\right] S_{0}
$$

made in the choice of standard contours $S_{0}$. Then the contour shape evolution can be written by combining eqn.(4) with eqn.(2) as :

$$
\tilde{S}(t)=\mathcal{R}(t) \Omega^{-1}\left(S_{0}(t)\right) \tilde{S}_{0}=\tilde{\mathcal{R}}(t) \tilde{S}_{0}(t)
$$

or

$$
\tilde{\mathcal{R}}(t)=\mathcal{R}(t) \Omega^{-1}\left(S_{0}(t)\right)
$$

The temporal change in the sequence of rigid motions can be written as:

$$
\frac{d \mathcal{R}}{d t}=\mathcal{R}\left(\mathcal{R}^{-1} \frac{d \mathcal{R}}{d t}\right) \equiv \mathcal{R} A
$$

where $A$ can be identified with the infinitesimal rotation arising from an infinitesimal deformation of $S_{0}(t)$. Eqn.(7) can be integrated to obtain the full motion for finite $t$ :

$$
\mathcal{R}\left(t_{2}\right)=\mathcal{R}\left(t_{1}\right) \mathcal{P} e^{\int_{t_{1}}^{t_{2}} A(t) d t}
$$

where $\mathcal{P}$ stands for the path ordered integral, the Wilson line integral $W$ :

$$
W_{21}=\mathcal{P} e^{\int_{t_{1}}^{t_{2}} A(t) d t}=1+\int_{t_{1}<t<t_{2}} A(t) d t+\int_{t_{1}<t<t^{\prime}<t_{2}} \int A(t) A\left(t^{\prime}\right) d t d t^{\prime}+\ldots
$$

in which the matrices are ordered such that the ones occurring at earlier times are on the left.

It can be seen from eqns.(5),(6) and (7), that $A$ transforms like a gauge potential:

$$
\tilde{A}=\Omega A \Omega^{-1}+\Omega \frac{d \Omega^{-1}}{d t}
$$

and the Wilson integral transforms as:

$$
\tilde{W}_{21}=\Omega_{1} W_{21} \Omega_{2}^{-1}
$$


Shapere and Wilczek exploited the invariance of (9) under rescaling of time, $t \rightarrow \tau(t)$, the measure scaling as $d t \rightarrow \dot{\tau} d t, A \rightarrow A / \dot{\tau}$, to rewrite it in a time-independent geometric form.

This was done $[5,6]$ by defining an abstract vector field $A$ on the tangent space to $S_{0}$. The projection $A(t)$ of $A$ at the contour shape $S_{0}(t)$ is evaluated in the direction $\frac{\delta S_{0}}{\delta t}$ in which the shape is changing:

$$
A(t) \equiv A_{\dot{S}_{0}}\left[S_{0}(t)\right]
$$

In terms of these projected vector fields, (8) was rewritten in a time-independent form for a given path and independent of the manner in which the path is parametrised in the contour shape space as:

$$
R\left(t_{2}\right)=R\left(t_{1}\right) \mathcal{P} e^{\int_{S_{0}\left(t_{1}\right)}^{S_{0}\left(t_{2}\right)} A\left(S_{0}\right) d S_{0}}
$$

Each of the components $A_{i}\left[S_{0}\right]$ of $A$ coming from each direction in the contour space generates a rigid motion and can be defined in terms of a fixed basis of tangent vectors $\left\{w_{i}\right\}$ at $S_{0}$ :

$$
A_{i}\left[S_{0}\right] \equiv A_{w_{i}}\left[S_{0}\right]
$$

An infinitesimal deformation $s(t)$ of a contour $S_{0}(t)$ can be represented as:

$$
S_{0}(t)=S_{0}+s(t)
$$

where an expansion of $s(t)$ can be made:

$$
s(t)=\sum_{i} \alpha_{i}(t) w_{i}
$$

It was shown in $[5,6]$ that for the particular case $S_{0}\left(t_{1}\right)=S_{0}\left(t_{2}\right)$, i,e., for a closed cycle in which the sequence of deformations returns the system to the original contour shape in its configuration space, the line integral in eqn.(9) becomes the closed Wilson loop which can be simplified to

$$
W=\mathcal{P} e^{\oint A(t) d t}=1+\frac{1}{2} \oint \sum_{i, j} F_{i j} \alpha_{i} \dot{\alpha}_{j} d t
$$

where

$$
F_{i j}=\frac{\partial A_{w_{i}}}{\partial w_{j}}-\frac{\partial A_{w_{j}}}{\partial w_{i}}+\left[A_{w_{i}}, A_{w_{j}}\right]
$$

The field strength tensor $F_{i j}$ gives the resultant net displacement when a sequence of successive deformations is made of $S_{0}$ around a closed path and is thus the curvature associated with the gauge potential.

In the configuration space of contour shapes, the orbit of each of the $n$ subsystems of the full coupled systems of oscillators undergoes the shape deformations described above. Because of the mutual couplings, 
the motion in phase space of any one oscillator coordinate is inseparably linked with that of any other phase space point which may be the coordinate of another oscillator. The deformation and motion in the configuration space of the various flow lines and closed paths of the entire coupled system can thus be viewed as those on the surface of a solid deformable body which is undergoing motion solely due to these deformations.

The full system of $n$ oscillators can be represented by an $n$-component vector $\psi^{i}, \quad(i=1, \ldots n)$ in an abstract complex vector space:

$$
\psi=\left(\begin{array}{l}
q_{1} \\
q_{2} \\
\cdot \\
\cdot \\
q_{n}
\end{array}\right)
$$

A rotation through an angle $\Lambda$ with respect to a chosen axis in this internal vector space does not change the state of the full system, but just takes one oscillator state $q_{i}$ to another:

$$
q_{i} \rightarrow \tilde{q}_{i}=U(\Lambda) q_{i}=e^{i t^{\alpha} \Lambda^{\alpha}} q_{i}
$$

where $t^{k}$ are $k$ number of $n \times n$ matrices and are representations of the generators of the transformation group. Each of the $q_{i}$ s represents the state of the $i$ th oscillator at time $t$.

There are $n$ independent gauge potentials $A_{w_{i}}$ corresponding to the $n$ independent internal rotations. Any two rotation matrices $U\left(\Lambda^{a}\right)$ and $U\left(\Lambda^{b}\right)$ do not commute unless $\Lambda^{a}$ and $\Lambda^{b}$ point in the same direction. On application of a common input to the full system, all the different $n$ oscillators respond to it. In this case emergence of a collective behaviour is determined by the same gauge potential, although perhaps by different amounts or strengths.

In the following section we would link these gauge potentials with the dynamically relevant variables of the coupled system. In section 4 we will attempt to understand the geometrical basis underlying the dynamics of phase synchronization between the oscillators. 


\section{COUPLED NONLINEAR OSCILLATORS SUBJECT TO FLUCTUATIONS}

We now consider the system of $n$ coupled nonlinear oscillators $q_{i}$ subject to additive Gaussian white noise $\xi_{i}$ in the limit of weak noise:

$$
\dot{q}_{i}=f_{i}\left(q_{1}, q_{2}, \ldots, q_{n}, \mu\right)+\xi_{i}, \quad(i=1, \ldots, n), \quad \mu \in R^{p}
$$

where the noise correlations are defined as:

$$
\left\langle\xi_{i}(t) \xi_{j}\left(t^{\prime}\right)\right\rangle=Q \delta_{i j} \delta\left(t-t^{\prime}\right)
$$

The eigenvalues of the linear stability matrix of the coupled deterministic system determine the route through which the full system moves towards a collective behaviour. A pure imaginary complex conjugate pair of eigenvalues at the bifurcation point with the remaining $(n-2)$ eigenvalues having nonzero real parts signals a Hopf bifurcation. The orbit structure near the nonhyperbolic fixed points $\left(q_{0}, \mu_{0}\right)$ of eqn.(1) is determined by the center manifold theorem. When the system described by (22) undergoes a change in stability through a Hopf bifurcation, one obtains a $p$-parameter family of vector fields on a 2-dimensional center manifold.

In this case one observes an emergent common frequency of oscillation for the coupled system. Such a situation automatically realises frequency synchronization also since the Hopf oscillator rotates with a characterisic frequency. If there are more than one Hopf bifurcations, clearly it indicates more than one common frequency of oscillation and one expects to observe a clustering of the various $n$ coupled oscillators around these common characteristic frequencies.

We aim to understand in this paper how phase synchronization results.

The full system of $\mathrm{n}$ oscillators changes stability as the parameter under consideration takes on different values, and at some parameter values undergoes bifurcations. We will study the system in the close neighborhood of the bifurcation points where the system exhibits critical behaviour. It is in these regimes that the behaviours of the individual oscillators gives way to the collective behaviour of the entire coupled system of the $\mathrm{n}$ oscillators. We employ center manifold reduction techniques for the system in the presence of fluctuations and perform a separation of variables in terms of fast and slow variables as in [10-11], exploiting their dynamical evolution on different time scales. A drastic simplification can then be made of the system's dynamics and one can write the probability $P\left(q_{i}, t\right)$ for the system to be in a certain configuration at time $t$ 
in the weak noise limit as the product:

$$
P\left(q_{i}, t\right)=p\left(q_{f} \mid q_{s}\right) P\left(q_{s}, t\right)
$$

where $q_{s}$ and $q_{f}$ are the slow and the fast variables respectively of the system. The probability $P\left(q_{s}, t\right)$ for the critical variables occurs on a slow time scale and is non-Gaussian in nature.

The properties of the fast variables depend upon the nonlinearities in the system. For instance in the case when the coupled system exhibits a cusp bifurcation the fast variable could exhibit non-Gaussian fluctuations (depending on the specific nonlinear interaction) as it is coupled to the critical variable. It can be shown [10] that the joint probability density $p\left(q_{f} \mid q_{s}\right)$ is confined to a narrow strip peaked about the center manifold. We are interested in the case when the coupled system also exhibits self-sustained oscillatory behaviour and makes a transition to limit cycle behaviour in the presence of fluctuations.

It was shown in $[10,11]$, that for a transition via a Hopf bifurcation, $p\left(q_{f} \mid q_{s}\right)$ has the time-independent Gaussian form in the $q_{f}$ variables with width which depends upon the slow variables $q_{s}$ :

$$
p\left(q_{f} \mid q_{s}\right)=\left(\frac{\sigma\left(q_{s}\right)}{\pi}\right)^{1 / 2} e^{-\sigma\left(q_{s}\right)\left(q_{f}-q_{f_{0}}\left(q_{s}\right)\right)^{2}}
$$

where the center manifold is obtained as a power series in $q_{s}: q_{f}=q_{f_{0}}\left(q_{s}\right)$. The center manifold theorem has been used in [12] for providing with a proof for the stability of the synchronised states. The enslaved stable modes are the fast variables which follow the dynamics of the center (critical) modes. We rewrite $f_{i}\left(q_{1}, q_{2}, \ldots, q_{n}, \mu_{i}\right)$ as

$$
f_{i}\left(q_{1}, q_{2}, \ldots, q_{n}, \mu_{i}\right)=-\frac{\delta F\left(q_{1}, q_{2}, \ldots, q_{n}, \mu_{i}\right)}{\delta q_{i}}
$$

The Fokker-Planck equation for the full system is:

$$
\frac{d P\left(q_{i}, t\right)}{d t}=\frac{d P\left(q_{s}, q_{f}, t\right)}{d t}=\frac{\partial}{\partial q_{i}}\left(P\left(q_{i}, t\right) \frac{\partial F}{\partial q_{i}}\right)+\frac{\partial^{2} P\left(q_{i}, t\right)}{\partial q_{i}^{2}}
$$

Using eqn.(23) we can rewrite this as

$$
\begin{aligned}
\frac{d P\left(q_{s}, q_{f}, t\right)}{d t} & =\frac{d}{d t}\left(p\left(q_{f} \mid q_{s}\right) P\left(q_{s}, t\right)\right)=\frac{d p\left(q_{f} \mid q_{s}\right)}{d t} P\left(q_{s}, t\right)+p\left(q_{f} \mid q_{s}\right) \frac{d P\left(q_{s}, t\right)}{d t} \\
& =-\left(H_{F P 1}\left(q_{f}, q_{s}\right)+H_{F P 2}\left(q_{s}, t\right)\right) P\left(q_{s}, q_{f}, t\right)
\end{aligned}
$$

Hence in the close proximity of the bifurcation, the operator $H_{F P}$ can be written in a separable form, the part $H_{F P}\left(q_{s}, t\right)$ independent of the fast variables. Here

$$
\left.-H_{F P 2}\left(q_{s}, t\right)\right) P\left(q_{s}, q_{f}, t\right)=\frac{\partial}{\partial q_{s}}\left(P\left(q_{i}, t\right) \frac{\partial F}{\partial q_{s}}\right)+\frac{\partial^{2} P\left(q_{i}, t\right)}{\partial q_{s}^{2}}
$$


We find it convenient to analyse the coupled dynamics in a path integral framework. We follow the procedure of Gozzi [13] to recast the system, eqns.(21),(27) as a path integral, and define:

$$
\Psi=P\left(q_{f}, q_{s}, t\right) e^{F\left(q_{i}\right) / 2}
$$

so that $(26),(28)$ can be rewritten as

$$
\frac{d \Psi}{d t}=-2 \mathcal{H}_{F P} \Psi
$$

where

$$
\mathcal{H}_{F P}=-\frac{1}{2} \frac{\partial^{2}}{\partial q_{i}^{2}}+\frac{1}{8}\left(\frac{\partial F}{\partial q_{i}}\right)^{2}-\frac{1}{4} \frac{\partial^{2} F}{\partial q_{i}^{2}}
$$

To enable computation of correlation functions within the path integral formalism, we introduce $n$ external sources $J_{i}$ to probe the full coupled system so that the partition function $Z[J]$ for the system can be written as the time ordered path integral

$$
Z[J]=\mathcal{N} \mathcal{T} \prod_{i} \int D q_{f} D q_{s} D \xi_{i} e^{-\frac{1}{Q} \int J_{i}\left(t^{\prime}\right) q_{i}\left(t^{\prime}\right) d t^{\prime}} P\left(q_{s}, t\right) p\left(q_{f} \mid q_{s}\right) \delta\left(q_{i}-q_{i \xi}\right) e^{-\int \frac{\xi_{i}^{2}}{4 Q} d t^{\prime}}
$$

where $q_{i \xi}$ denote the solution of the system of Langevin equations (21), $\mathcal{T}$ denotes time ordering and $\mathcal{N}$ is the normalization constant. From eqns.(21) one can write

$$
\delta\left(q_{i}-q_{i \xi}\right)=\delta\left(\dot{q}_{i}-f_{i}\left(q_{1}, q_{2}, \ldots, q_{n}\right)-\xi_{i}\right)\left\|\frac{\delta \xi_{i}}{\delta q_{i}}\right\|
$$

We can rewrite the Jacobian $\left\|\frac{\delta \xi_{i}}{\delta q_{i}}\right\|$ of the transformation $\xi_{i} \rightarrow q_{i}$ as

$$
\begin{aligned}
\left\|\frac{\delta \xi_{i}}{\delta q_{i}}\right\| & =\operatorname{det}\left[\left(\delta_{i j} \partial_{t}-\frac{\partial f_{i}\left(q_{1}, q_{2}, \ldots, q_{n}\right)}{\partial q_{j}\left(t^{\prime}\right)}\right) \delta\left(t-t^{\prime}\right)\right] \\
& =\exp \left\{\operatorname{tr} \ln \partial_{t}\left(\delta_{i j} \delta\left(t-t^{\prime}\right)-\partial_{t^{\prime}}{ }^{-1} \frac{\partial f_{i}}{\partial q_{j}\left(t^{\prime}\right)}\right)\right\}
\end{aligned}
$$

The operator $\partial_{t^{\prime}}{ }^{-1}$ satisfies the relation

$$
\partial_{t} G\left(t-t^{\prime}\right)=\delta\left(t-t^{\prime}\right)
$$

Then we can rewrite eqn.(34) in terms of the Green function in (35) as:

$$
\left\|\frac{\delta \xi_{i}}{\delta q_{j}}\right\|=\exp \left\{\operatorname{tr}\left[\ln \partial_{t}+\ln \left(\delta\left(t-t^{\prime}\right)+G_{i j}\left(t-t^{\prime}\right) \frac{\partial f_{i}}{\partial q_{j}\left(t^{\prime}\right)}\right)\right]\right\}
$$

The system evolves forward in time. Hence

$$
G\left(t-t^{\prime}\right)=\theta\left(t-t^{\prime}\right)
$$


Using this and expanding the logarithm in the argument of the exponential, we can simplify eqn.(36) to

$$
\left\|\frac{\delta \xi_{i}}{\delta q_{j}}\right\|=e^{\operatorname{tr} \ln \partial_{t}} e^{\int_{0}^{t} d t^{\prime} \theta(0) \frac{\partial f_{i}}{\partial q_{j}\left(t^{\prime}\right)}}
$$

Substituting this back into eqn.(32) and using the mid-point prescription $\theta(0)=1 / 2$ of Stratonovich, we have

$$
Z[J]=\mathcal{N} \mathcal{T} \prod_{i} \int D q_{f} D q_{s} D \xi_{i} e^{-\frac{1}{Q} \int_{0}^{t} J_{i}\left(t^{\prime}\right) q_{i}\left(t^{\prime}\right) d t^{\prime}} e^{\frac{1}{2} \int_{0}^{t} \frac{\partial f_{i}}{\partial q_{j}\left(t^{\prime}\right)}} e^{-\frac{1}{4 Q} \int_{0}^{t} d t^{\prime}\left(\dot{q}_{i}-f_{i}\left(q_{1}, q_{2}, \ldots, q_{n}\right)\right)^{2}}
$$

Eqn.(39) can be reduced to

$$
\begin{aligned}
Z[J] & =\mathcal{N} \mathcal{T} \prod_{i} \int D q_{f} D q_{s} e^{-\frac{1}{Q} \int_{0}^{t} J_{i}\left(t^{\prime}\right) q_{i}\left(t^{\prime}\right) d t^{\prime}} e^{-\int_{0}^{t} d t^{\prime}\left[\frac{1}{2} \frac{\partial^{2} F}{\partial q_{i} \partial q_{j}}+\frac{1}{4 Q} \dot{q}_{i}^{2}+\frac{1}{4 Q}\left(\frac{\partial F}{\partial q_{i}}\right)^{2}\right]} e^{-\frac{1}{2 Q}(F(t)-F(0))} \\
& =\mathcal{N} \mathcal{T} \prod_{i} \int D q_{f} D q_{s} e^{-\int_{0}^{t} d t^{\prime}\left[\mathcal{L}^{F P}+\frac{1}{Q} J_{i}\left(t^{\prime}\right) q_{i}\left(t^{\prime}\right)\right.} e^{-\frac{1}{2 Q}(F(t)-F(0))}
\end{aligned}
$$

where we have defined a Fokker-Planck lagrangian

$$
\mathcal{L}^{F P}\left(q_{i}, \dot{q}_{i}, t\right)=\frac{1}{4 Q} \dot{q}_{i}^{2}+\frac{1}{4 Q}\left(\frac{\delta F}{\delta q_{i}}\right)^{2}+\frac{1}{2} \frac{\delta^{2} F}{\delta q_{i} \delta q_{j}}=\frac{1}{4 Q} \dot{q}_{i}^{2}+f_{i}^{2}-\frac{1}{2} \frac{\delta f_{j}}{\delta q_{i} \delta q_{i}}
$$

which is related to the Fokker-Planck hamiltonian $\mathcal{H}_{F P}$ defined in eqns.(30),(31) through a Legendre transformation:

$$
\mathcal{H}_{F P}\left(\pi_{i}, q_{i}, t\right)=\pi_{i} \dot{q}_{i}-\mathcal{L}^{F P}\left(q_{i}, \dot{q}_{i}, t\right)
$$

Here $\pi_{i}$ are the momenta canonically conjugate to the variables $q_{i}$ :

$$
\frac{\delta \mathcal{L}^{F P}}{\delta \dot{q}_{i}}=\pi_{i}=\frac{1}{Q} \dot{q}_{i}
$$

so that

$$
\mathcal{H}_{F P}\left(\pi_{i}, q_{i}, t\right)=Q \pi_{i}^{2}+\frac{1}{4 Q}\left(\frac{\delta F}{\delta q_{i}}\right)^{2}+\frac{1}{2} \frac{\delta^{2} F}{\delta q_{i} \delta q_{j}}
$$

We use these relations in eqn.(40) to write the partition function as

$$
Z[J]=\mathcal{N} \mathcal{T} \prod_{i} \int D \pi_{i} D q_{i} e^{-\int_{0}^{t} d t^{\prime}\left[\mathcal{H}_{F P}\left(\pi_{i}, q_{i}, t\right)+\frac{1}{Q} J_{i}\left(t^{\prime}\right) q_{i}\left(t^{\prime}\right)\right]}
$$

From eqns.(27),(29),(30) and (31), we see that in the close proximity of the bifurcation $\mathcal{H}_{F P}$ can be written in a separable form as

$$
\mathcal{H}_{F P}\left(\pi_{i}, q_{i}, t\right)=\mathcal{H}_{F P}\left(\pi_{f}, q_{f} ; \pi_{s}, q_{s}, t\right)=\mathcal{H}_{F P 1}\left(\pi_{f}, q_{f} ; q_{s}, t\right)+\mathcal{H}_{F P 2}\left(\pi_{s}, q_{s}, t\right)
$$

Thus the corresponding $\mathcal{L}^{F P}$ can also be split up as $\quad \mathcal{L}^{F P}\left(q_{i}, \dot{q}_{i}, t\right)=\mathcal{L}_{1}^{F P}\left(q_{f}, \dot{q}_{f}, t\right)+\mathcal{L}_{2}^{F P}\left(q_{s}, \dot{q}_{s}, t\right)$. Then we can write

$$
Z[J]=\mathcal{N} \mathcal{T} \int D q_{f} D q_{s} e^{-\int_{0}^{t} d t^{\prime}\left[\mathcal{L}_{1}^{F P}\left(q_{f}, \dot{q}_{f}\right)+\mathcal{L}_{2}^{F P}\left(q_{s}, \dot{q}_{s}, t\right)+\frac{1}{Q} J_{i}\left(t^{\prime}\right) q_{i}\left(t^{\prime}\right)\right]}
$$


An averaging over the fast degrees of freedom enables the partition function to be written in terms of an effective Lagrangian as a function of only the slow degrees of freedom. This can be done by first rewriting the fast degrees of freedom in action-angle variables $(\theta, I)$. The emergence of a non Abelian gauge structure can then be seen arising from the evolution of the slow dynamics but induced by the fast variables. After tracing the origin of the induced gauge potential to the slow dynamics, we obtain the conditions necessary to be satisfied in order for the coupled elements to be synchronised in phase.

To begin with, we introduce a generating function $S^{(\alpha)}\left(q_{f}, I ; q_{s}\right)$ which effects the transformation $\left(q_{f}, \pi_{f}\right) \rightarrow$ $(\theta, I)$ to the action angle variables:

$$
\frac{\partial S^{(\alpha)}\left(q_{f}, I ; q_{s}\right)}{\partial q_{i}}=\pi_{i} \quad ; \quad \frac{\partial S^{(\alpha)}\left(q_{f}, I ; q_{s}\right)}{\partial I_{i}}=\theta_{i}
$$

$S^{(\alpha)}\left(q_{f}, I ; q_{s}\right)$ is many-valued and time dependent since the slow variables change with time.

The phase space structure associated with adiabatic holonomy in classical systems was studied by Gozzi and Thacker [14] through Hamiltonian dynamics. We find it useful to employ their methods for our study of coupled oscillatory systems in a fluctuating environment. Denote $\mathcal{H}_{1}\left(I, q_{s}, t\right)=\mathcal{H}_{F P 1}\left(q_{f}\left(\theta, I, q_{s}\right), \pi_{f}\left(\theta, I, q_{s}\right), t\right)$. Using the canonical transformation law, $\mathcal{H}_{1}$ can be expressed in terms of the action-angle variables as:

$$
\bar{H}_{1}\left(\theta, I, q_{s}(t)\right)=\mathcal{H}_{1}\left(I, q_{s}, t\right)+\dot{q}_{s l} \frac{\partial S^{(\alpha)}\left(q_{f}, I ; q_{s}\right)}{\partial q_{s l}}
$$

Using the methods of [14] and [15-17], we determine the dynamics of the $M$ critical slow variables $q_{s}$ of the system by averaging out the $N$ fast variables which influence them:

$$
\begin{aligned}
\left\langle\left\langle\bar{H}_{1}\right\rangle\right\rangle & =\frac{1}{(2 \pi)^{N}} \int d^{N} \theta \bar{H}_{1}\left(\theta, I, q_{s}(t)\right) \\
& =\frac{1}{(2 \pi)^{N}} \int d^{N} \theta\left(\mathcal{H}_{1}\left(I, q_{s}\right)+\dot{q}_{s l} \frac{\partial S^{(\alpha)}\left(q_{f}, I ; q_{s}\right)}{\partial q_{s l}}\right)
\end{aligned}
$$

where the double angular brackets denote the averaging over all $\theta: \quad\langle\langle f\rangle\rangle=\frac{1}{(2 \pi)^{N}} \int d^{N} \theta f$. Since $S^{(\alpha)}\left(q_{f}, I ; q_{s}\right)$ is multi-valued, the single-valued function

$$
\zeta\left(\theta, I, q_{s}\right)=S^{(\alpha)}\left(q_{f}\left(\theta, I, q_{s}\right), \pi_{s}\left(\theta, I, q_{s}\right), q_{s}\right) \quad, \quad(0 \leq \theta \leq 2 \pi)
$$

is introduced [18]. We have

$$
\frac{\partial \zeta}{\partial q_{s l}}=\frac{\partial S^{\alpha}}{\partial q_{s l}}+\pi_{f_{i}} \frac{\partial q_{f_{i}}}{\partial q_{s l}}
$$

Hence this can be substituted into eqn.(50) to obtain

$$
\left\langle\left\langle\bar{H}_{1}\right\rangle\right\rangle=\mathcal{H}_{1}\left(I, q_{s}\right)+\dot{q}_{s l}\left\langle\left\langle\frac{\partial \zeta}{\partial q_{s l}}-\pi_{f_{i}} \frac{\partial q_{f_{i}}}{\partial q_{s l}}\right\rangle\right\rangle
$$


The total Hamiltonian of the system is given, after performing the angle averages by:

$$
\begin{aligned}
H_{a v}\left(I, \pi_{s}, q_{s}\right) & =\left\langle\left\langle\mathcal{H}_{1}\left(q_{f}, \pi_{f} ; q_{s}\right)+\mathcal{H}_{2}\left(q_{s}, \pi_{s}\right)\right\rangle\right\rangle=\left\langle\left\langle\bar{H}_{1}\left(\theta, I ; q_{s}\right)+\mathcal{H}_{2}\left(q_{s}, \pi_{s}\right)\right\rangle\right\rangle \\
& =\bar{H}\left(I, \pi_{s}, q_{s}\right)+\dot{q}_{s l}\left\langle\left\langle\frac{\partial \zeta}{\partial q_{s l}}-\pi_{f_{i}} \frac{\partial q_{f_{i}}}{\partial q_{s l}}\right\rangle\right\rangle
\end{aligned}
$$

where we have let

$$
\bar{H}\left(I, \pi_{s}, q_{s}\right)=\mathcal{H}_{1}\left(I, q_{s}\right)+\mathcal{H}_{2}\left(q_{s}, \pi_{s}\right)
$$

The Gibbs partition function in eqn.(45) can be rewritten in terms of the fast and slow variables as

$$
\begin{aligned}
Z[J] & =\mathcal{N} \mathcal{T} \int D \pi_{f} D \pi_{s} D q_{f} D q_{s} e^{-\int_{0}^{t} d t^{\prime}\left[\mathcal{H}_{F P}\left(q_{f}, \pi_{f} ; q_{s}\right)+\mathcal{H}_{F P_{2}}\left(q_{s}, \pi_{s}\right)+\frac{1}{Q}\left(J_{s}\left(t^{\prime}\right) q_{s}\left(t^{\prime}\right)+J_{f}\left(t^{\prime}\right) q_{f}\left(t^{\prime}\right)\right)\right]} \\
& =\mathcal{N} \mathcal{T} \int D \pi_{s} D q_{s} D I D \theta e^{-\int_{0}^{t} d t^{\prime}\left[\mathcal{H}_{1}\left(I, q_{s}\right)+\mathcal{H}_{2}\left(q_{s}, \pi_{s}\right)-\dot{q}_{s l} \pi_{f_{i}} \frac{\partial q_{f}}{\partial q_{s l}}+\dot{q}_{s l} \frac{\partial \zeta}{\partial q_{s l}}+\frac{1}{Q}\left(J_{s}\left(t^{\prime}\right) q_{s}\left(t^{\prime}\right)+J_{f}\left(t^{\prime}\right) \theta\left(t^{\prime}\right)\right)\right]} \\
& =\mathcal{N} \mathcal{T} \int D \pi_{s} D q_{s} D I D \theta e^{-\int_{0}^{t} d t^{\prime}\left[\bar{H}\left(I, \pi_{s}, q_{s}\right)-\dot{q}_{s l} \pi_{f} \frac{\partial q_{f}}{\partial q_{s l}}+\frac{1}{Q}\left(J_{s}\left(t^{\prime}\right) q_{s}\left(t^{\prime}\right)+J_{f}\left(t^{\prime}\right) \theta\left(t^{\prime}\right)\right)\right]}
\end{aligned}
$$

Performing the $\theta$ integration and simplifying the resulting expression, we get

$$
Z[J] \approx \mathcal{N} \mathcal{T} \int D \pi_{s} D q_{s} D I e^{-\int_{0}^{t} d t^{\prime}\left(H_{a v}\left(I, \pi_{s}, q_{s}\right)+\frac{1}{Q} J_{s} q_{s}\right)}
$$

We use the Magnus expansion [19,20,21] for expanding the time-ordered integral, which gives the final state properties in terms of integrals over the initial state ones, to rewrite eqn.(57) as:

$$
\begin{aligned}
Z[J] & \approx \mathcal{N} \int D \pi_{s} D q_{s} D I \exp \left\{-\int_{0}^{t} d t^{\prime}\left(H_{a v}+\frac{1}{Q} J_{s} q_{s}+\frac{1}{2}\left[H_{a v}\left(t^{\prime}\right), \int_{0}^{t^{\prime}} H_{a v}\left(t_{1}\right) d t_{1}\right]\right.\right. \\
& +\frac{1}{4}\left[H_{a v}\left(t^{\prime}\right), \int_{0}^{t^{\prime}}\left[H_{a v}\left(t_{2}\right), \int_{0}^{t_{2}} H_{a v}\left(t_{1}\right) d t_{1}\right] d t_{2}\right]+\frac{1}{12}\left[\left[H_{a v}\left(t^{\prime}\right), \int_{0}^{t^{\prime}} H_{a v}\left(t_{2}\right) d t_{2}\right], \int_{0}^{t^{\prime}} H_{a v}\left(t_{1}\right) d t_{1}\right] \\
& +\ldots)\}
\end{aligned}
$$

The variation of parameter $(\mathrm{s}) \mu_{i}$ in time of the system brings about change in its stability. The commutator terms in the Magnus expansion hence arise on account of this parametric time dependence of the Hamiltonian: $\left[H_{a v}\left(t^{\prime}\right), H_{a v}\left(t_{1}\right)\right]=\left[H_{a v}\left(\mu_{i}\left(t^{\prime}\right)\right), H_{a v}\left(\mu_{j}\left(t_{1}\right)\right)\right]$.

The necessity of the time ordering is also motivated by the work of ref.[22] who have shown that the order of arrival of signals at an oscillator in a network of pulse-coupled oscillators is crucial in determining changes in its phase.

Retaining terms only upto the first commutator in the expansion and substituting for $H_{a v}$ from eqn.(54), we obtain after some simplifications:

$$
Z[J] \approx \mathcal{N} \int D \pi_{s} D q_{s} D I \exp \left\{-\int_{0}^{t} d t^{\prime}\left(\bar{H}\left(I, \pi_{s}, q_{s}\right)+\dot{q}_{s_{l}}\left\langle\left\langle\frac{\partial \zeta}{\partial q_{s l}}\right\rangle\right\rangle-\dot{q}_{s_{l}}\left\langle\left\langle\pi_{f_{i}} \frac{\partial q_{f_{i}}}{\partial q_{s l}}\right\rangle\right\rangle+\frac{1}{Q} J_{s} q_{s}\right.\right.
$$




$$
\begin{aligned}
& -\frac{1}{2}\left[\bar{H}\left(I, \pi_{s}, q_{s}, t^{\prime}\right), \int_{0}^{t^{\prime}} \bar{H}\left(I, \pi_{s}, q_{s}, t_{1}\right) d t_{1}\right]+\frac{1}{2}\left[\bar{H}\left(I, \pi_{s}, q_{s}, t^{\prime}\right), \int_{0}^{t^{\prime}} d t_{1} \dot{q}_{s_{m}}\left\langle\left\langle\pi_{f_{k}} \frac{\partial q_{f_{k}}}{\partial q_{s_{m}}}\right\rangle\right\rangle\right] \\
& \left.+\frac{1}{2}\left[\dot{q}_{s_{m}}\left\langle\left\langle\pi_{f_{k}} \frac{\partial q_{f_{k}}}{\partial q_{s_{m}}}\right\rangle\right\rangle, \int_{0}^{t^{\prime}} \bar{H}\left(I, \pi_{s}, q_{s}, t_{1}\right) d t_{1}\right]-\frac{1}{2}\left[\dot{q}_{s_{l}}\left\langle\left\langle\pi_{f_{i}} \frac{\partial q_{f_{i}}}{\partial q_{s l}}\right\rangle\right\rangle, \int_{0}^{t^{\prime}} d t_{1} \dot{q}_{s_{m}}\left\langle\left\langle\pi_{f_{k}} \frac{\partial q_{f_{k}}}{\partial q_{s_{m}}}\right\rangle\right\rangle\right]\right\}
\end{aligned}
$$

From here we can define an effective Hamiltonian $H_{\text {eff }}$ :

$$
H_{e f f}=\bar{H}\left(I, \pi_{s}, q_{s}\right)+\dot{q}_{s_{l}}\left\langle\left\langle\frac{\partial \zeta}{\partial q_{s l}}\right\rangle\right\rangle-\dot{q}_{s_{l}}\left\langle\left\langle\pi_{f_{i}} \frac{\partial q_{f_{i}}}{\partial q_{s l}}\right\rangle\right\rangle-\frac{1}{2}\left[\dot{q}_{s_{l}}\left\langle\left\langle\pi_{f_{i}} \frac{\partial q_{f_{i}}}{\partial q_{s_{l}}}\right\rangle\right\rangle, \int_{0}^{t^{\prime}} d t_{1} \dot{q}_{s_{m}}\left\langle\left\langle\pi_{f_{k}} \frac{\partial q_{f_{k}}}{\partial q_{s_{m}}}\right\rangle\right\rangle\right]
$$

From a Hamiltonian variational principle, it was shown in [14] from simple arguments that the averaged fast motion induces an effective gauge field which acts on the slow variables. We follow these arguments closely for the coupled system subject to fluctuations near the instability. The variational principle gives:

$$
\begin{aligned}
\delta S_{e f f} & =\delta \int_{0}^{T} d t\left[\pi_{s l} \dot{q}_{s l}-H_{e f f}\left(I, \pi_{s}, q_{s}\right)\right]=0 \\
& =\delta \int_{0}^{T} d t\left\{\pi_{s l} \dot{q}_{s l}-\bar{H}\left(I, \pi_{s}, q_{s}\right)-\dot{q}_{s l}\left\langle\left\langle\frac{\partial \zeta}{\partial q_{s l}}-\pi_{f_{i}} \frac{\partial q_{f_{i}}}{\partial q_{s l}}\right\rangle\right\rangle-\frac{1}{2}\left[\dot{q}_{s_{l}}\left\langle\left\langle\pi_{f_{i}} \frac{\partial q_{f_{i}}}{\partial q_{s l}}\right\rangle\right\rangle, \int_{0}^{t^{\prime}} d t_{1} \dot{q}_{s_{m}}\left\langle\left\langle\pi_{f_{k}} \frac{\partial q_{f_{k}}}{\partial q_{s m}}\right\rangle\right\rangle\right]\right\}=0 \\
& =\delta \int_{0}^{T} d t\left\{\left[\pi_{s l}+\left\langle\left\langle\pi_{f_{i}} \frac{\partial q_{f_{i}}}{\partial q_{s l}}\right\rangle\right\rangle\right] \dot{q}_{s l}-\bar{H}\left(I, \pi_{s}, q_{s}\right)+\frac{1}{2}\left[\dot{q}_{s_{l}}\left\langle\left\langle\pi_{f_{i}} \frac{\partial q_{f_{i}}}{\partial q_{s l}}\right\rangle\right\rangle, \int_{0}^{t^{\prime}} d t_{1} \dot{q}_{s_{m}}\left\langle\left\langle\pi_{f_{k}} \frac{\partial q_{f_{k}}}{\partial q_{s m}}\right\rangle\right\rangle\right]\right\}
\end{aligned}
$$

The term having the single-valued function $\zeta$ vanishes since it is a total time derivative.

Varying $S_{\text {eff }}$ with respect to $\pi_{s}$ and $q_{s}$, keeping the end-points fixed gives:

$$
\begin{aligned}
\delta S_{e f f} & =\int_{0}^{T} d t\left\{\delta \pi_{s l}\left(\dot{q}_{s l}-\frac{\partial \bar{H}}{\partial \pi_{s l}}\right)+\delta q_{s l}\left[\left(\frac{\partial}{\partial q_{s l}}\left\langle\left\langle\pi_{f_{i}} \frac{\partial q_{f_{i}}}{\partial q_{s_{m}}}\right\rangle\right\rangle-\frac{\partial}{\partial q_{s_{m}}}\left\langle\left\langle\pi_{f_{i}} \frac{\partial q_{f_{i}}}{\partial q_{s l}}\right\rangle\right\rangle+\frac{1}{2}\left[\left\langle\left\langle\pi_{f_{i}} \frac{\partial q_{f_{i}}}{\partial q_{s_{m}}}\right\rangle\right\rangle,\left\langle\left\langle\pi_{f k} \frac{\partial q_{f_{k}}}{\partial q_{s l}}\right\rangle\right\rangle\right]\right) \dot{q}_{s_{m}}\right.\right. \\
& \left.\left.-\frac{\partial \bar{H}}{\partial q_{s l}}-\dot{\pi}_{s l}\right]\right\}
\end{aligned}
$$

We define as in [14], the quantity in angular brackets as

$$
\left\langle\left\langle\pi_{f_{i}} \frac{\partial q_{f_{i}}}{\partial q_{s l}}\right\rangle\right\rangle=A_{l}
$$

Then $\delta S_{\text {eff }}=0 \quad$ leads to

$$
\begin{aligned}
\dot{q}_{s l} & =\frac{\partial \bar{H}}{\partial \pi_{s l}} \\
\dot{\pi}_{s l} & =-\frac{\partial \bar{H}}{\partial q_{s l}}+\left(\frac{\partial A_{m}}{\partial q_{s l}}-\frac{\partial A_{l}}{\partial q_{s m}}+\frac{1}{2}\left[A_{l}, A_{m}\right]\right) \dot{q}_{s_{m}}
\end{aligned}
$$

As in [14] we can identify $A_{l}$ with a gauge potential, and a curvature tensor $\mathcal{F}_{l m}$ can be defined as

$$
\mathcal{F}_{l m}=\frac{\partial A_{m}}{\partial q_{s l}}-\frac{\partial A_{l}}{\partial q_{s_{m}}}+\frac{1}{2}\left[A_{l}, A_{m}\right]
$$

so that the momenta in (64) can be rewritten as

$$
\dot{\pi}_{s l}=-\frac{\partial \bar{H}}{\partial q_{s l}}+\mathcal{F}_{l m} \frac{\partial \bar{H}}{\partial \pi_{s l}}
$$


The commutator terms in the momenta and curvature tensor were absent in ref.[14] since the Magnus expansion for the time ordered integral was not used there.

It should be noted that these commutator terms in eqns.(61), (62),(64)-(66) arising from terms such as $\left[H(\mu), H\left(\mu^{\prime}\right)\right]$ in eqn.(58) could in general impart a non-flat nature to the connection and generate a curvature which is non-trivial by effectively generating new parameters which were not present in the original Hamiltonian. (See for example $[23,24]$ where in the context of examining the connection between classical and quantum anholonomy for some interesting systems (in particular the displaced harmonic oscillator [25]), it was shown that for time-varying Hamiltonians, the original Hamiltonian must be embedded into a larger class for locating the effective parameter space where the Berry phase two-form has singularities). The appearance of a nontrivial gauge structure in general dynamical systems, including classical systems, due to a slow variation of the parameters was also explicitly demonstrated in the seminal work in [26].

Eqn.(64) shows that the curvature tensor $\mathcal{F}_{l m}$ exerts a velocity dependent force on the slow variables. In order to write canonical equations of motion, one has to therefore introduce modified Poisson bracket relations in the slow-variable space:

$$
\left\{f\left(q_{s}, \pi_{s}\right), g\left(q_{s}, \pi_{s}\right)\right\}=\left\{\frac{\partial f}{\partial \pi_{s l}} \frac{\partial g}{\partial q_{s l}}-\frac{\partial f}{\partial q_{s l}} \frac{\partial g}{\partial \pi_{s l}}\right\}-\mathcal{F}_{l m} \frac{\partial f}{\partial \pi_{s l}} \frac{\partial g}{\partial \pi_{s m}}
$$

Thus the gauge potential coupled to the slow variables is induced by the fast degrees of freedom as is evident from (63), the spontaneous appearance of the gauge symmetry being associated with the phase degrees of freedom of the center modes.

The emergence of a gauge structure for the system follows from the crucial property of separability of the variables as slow and fast ones evolving at different time scales, which results from the slaving principle for the stable modes near the bifurcation in a noisy system. This leads to the motion and deformation of the closed orbits in the configuration space. The rotational symmetries of the sequence of successive deformations of each orbit brought about the gauge potential discussed in Section 2. The analysis above in the current section shows that this can be related to the dynamically evolving variables of the coupled system.

Having made the correspondence of the gauge potential $A_{l}$ and the curvature tensor $\mathcal{F}_{l m}$ with the dynamics of the actual coupled system through the fast and slow variables, we proceed to examine under what conditions phase locked behaviour and full synchronization would occur in a coupled system. 


\section{CONDITION FOR SYNCHRONIZATION BETWEEN THE COUPLED OSCILLATORS}

At any instant of time, the phase difference between two oscillators $q_{1}$ and $q_{2}$ located at two different points $x$ and $y$ in the configuration space can be found from their inner product:

$$
\begin{aligned}
\cos \theta_{y} & =\frac{\left(q_{2}(y), q_{1}(y)\right)}{\left|\left(q_{2}(y), q_{1}(y)\right)\right|} \\
& =\int d^{d} y d^{d} x \operatorname{Tr}\left(P\left(e^{\int_{x}^{y} A_{\mu}^{\alpha}(s) t^{\alpha} d s_{\mu}}\right)\right) \frac{\left(q_{2}(y), q_{1}(x)\right)}{\left|\left(q_{2}(y), q_{1}(y)\right)\right|}
\end{aligned}
$$

where $\theta_{y}$ denotes the angle between the oscillators $q_{1}(x)$ and $q_{2}(y)$ in configuration space, measured at the coordinate $y$. The path-ordered Wilson line integral appears in the equation above since $q_{1}(x)$ must be parallelly transported to the coordinate point $y$ in order to compare it with $q_{2}$ located at $y . P$ denotes the path-ordering. Since the $q_{i}$ s are related to each other through a gauge transformation in the $n$-dimensional configuration space, this can be rewritten using eqn.(20) as

$$
\begin{aligned}
\cos \theta_{y} & =\int d^{d} y d^{d} x \operatorname{Tr}\left(e^{-i t^{\beta} \Lambda^{\beta}} P\left(e^{\int_{x}^{y} A_{\mu}^{\alpha}(s) t^{\alpha} d s_{\mu}}\right)\right) \frac{\left(q_{1}(y), q_{1}(x)\right)}{\left|\left(q_{2}(y), q_{1}(y)\right)\right|} \\
& =\int d^{d} y d^{d} x \operatorname{Tr}\left(e^{-i t^{\beta} \Lambda^{\beta}} P\left(e^{\int_{x}^{y} A_{\mu}^{\alpha}(s) t^{\alpha} d s_{\mu}}\right) P\left(e^{\int_{y}^{x} A_{\lambda}^{\gamma}(p) t^{\gamma} d p_{\lambda}}\right)\right) \frac{\left(q_{1}(y), q_{1}(y)\right)}{\left|\left(q_{2}(y), q_{1}(y)\right)\right|} \\
& =\int d^{d} y d^{d} x \operatorname{Tr}\left(e^{-i t^{\beta} \Lambda^{\beta}} P\left(e^{\int_{x}^{y} A_{\mu}^{\alpha}(s) t^{\alpha} d s_{\mu}}\right) P\left(e^{\int_{x}^{y} A_{\lambda}^{\gamma}(p) t^{\gamma} d p_{\lambda}}\right)\right) \frac{1}{\left|\left(q_{2}(y), q_{1}(y)\right)\right|}
\end{aligned}
$$

since $\left(q_{1}(y), q_{1}(y)\right)=1$. If the angle between $q_{1}$ and $q_{2}$ remains constant for all times, then the oscillators $q_{1}$ and $q_{2}$ would be phase-locked; if the angle between them is vanishing for all time, the oscillators would be fully synchronized in phase with each other.

We would like to determine the conditions under which the phases of any two oscillators in a coupled nonlinear system would be locked and fully synchronised. Since each $q_{i}$ is an oscillator, each undergoes periodic dynamics in the configuration space.

Let $q_{1}(y)$ after being parallelly transported from coordinate $x$, now return to the point $x$ during the course of its temporal evolution. We denote the state of this oscillator after completing one orbit and returning to $x$ by $q_{1}^{\prime}(x)$. By the time $q_{1}$ completes this orbit, $q_{2}$ would have evolved to another point $z$. Hence we would now like to calculate the angle between $q_{2}(z)$ and $q_{1}^{\prime}(x)$.

We have:

$$
q_{1}^{\prime}(x)=P\left(e^{\oint A_{\mu}^{\alpha}(s) t^{\alpha} d s_{\mu}}\right) q_{1}(x)
$$


$q_{2}(z)$ can be parallelly transported to $x$ to compare it with $q_{1}^{\prime}(x)$ :

$$
q_{2}(x)=P\left(e^{\int_{z}^{x} A_{\mu}^{\alpha}(s) t^{\alpha} d s_{\mu}}\right) q_{2}(z)
$$

Then the angle between $q_{1}$ and $q_{2}$ at $x$ can be calculated:

$$
\begin{aligned}
\cos \theta_{x} & =\frac{\left(q_{2}(x), q_{1}^{\prime}(x)\right)}{\left|\left(q_{2}(x), q_{1}^{\prime}(x)\right)\right|} \\
& =\int d^{d} z d^{d} x \operatorname{Tr}\left(\left(P\left(e^{\int_{z}^{x} A_{\mu}^{\alpha}(s) t^{\alpha} d s_{\mu}}\right)\right)^{T} P\left(e^{\oint A_{\nu}^{\beta}(p) t^{\beta} d p_{\nu}}\right)\right) \frac{\left(q_{2}(z), q_{1}(x)\right)}{\left|\left(q_{2}(x), q_{1}^{\prime}(x)\right)\right|} \\
& =\int d^{d} z d^{d} x \operatorname{Tr}\left(\left(P\left(e^{\int_{z}^{x} A_{\mu}^{\alpha}(s) t^{\alpha} d s_{\mu}}\right) e^{i t^{\beta} \Lambda^{\beta}}\right)^{T} P\left(e^{\oint A_{\nu}^{\beta}(p) t^{\beta} d p_{\nu}}\right)\right) \frac{\left(q_{1}(z), q_{1}(x)\right)}{\left|\left(q_{2}(x), q_{1}^{\prime}(x)\right)\right|} \\
& =\int d^{d} z d^{d} x \operatorname{Tr}\left(\left(P\left(e^{\int_{z}^{x} A_{\mu}^{\alpha}(s) t^{\alpha} d s_{\mu}}\right) e^{i t^{\beta} \Lambda^{\beta}} P\left(e^{\int_{x}^{z} A_{\kappa}^{\alpha}(l) t^{\alpha} d l_{\kappa}}\right)\right)^{T} P\left(e^{\oint A_{\nu}^{\beta}(p) t^{\beta} d p_{\nu}}\right)\right) \frac{\left(q_{1}(x), q_{1}(x)\right)}{\left|\left(q_{2}(x), q_{1}^{\prime}(x)\right)\right|}(72)
\end{aligned}
$$

The change in the angle between $q_{1}$ and $q_{2}$ during a time interval $t$ can be found using eqns.(69) and (72) and by simplifying the resulting expression, to lowest order in $\Lambda$ to be

$\cos \theta_{y}-\cos \theta_{x}=-\frac{C(p)}{2} \int d^{d} x d^{d} y\left\{-i \Lambda^{a} F_{i j}^{a}+F_{i j}^{a} \int_{y}^{x} A_{\mu}^{a}(s) d s_{\mu}+\left(\delta^{\alpha a}+\frac{\epsilon^{\beta \alpha a}}{2} \Lambda^{\beta}\right) F_{i j}^{a} \int_{y}^{x} A_{\mu}^{\alpha}(s) d s_{\mu}+\ldots\right\}$

In arriving at this expression we have used the relation for the Wilson loop integral

$$
P\left(e^{\oint A_{\mu}^{\beta}(s) t^{a} d s_{\mu}}\right)=e^{F_{\mu \nu}}
$$

in which the $t^{a}$ (introduced earlier in eqn.(20)) are generators of the Lie algebra

$$
\left[t^{a}, t^{b}\right]=i \epsilon^{a b c} t^{c}
$$

and

$$
t^{a} F_{\mu \nu}^{a}=\partial_{\mu} t^{a} A_{\nu}^{a}-\partial_{\nu} t^{a} A_{\mu}^{a}-i\left[t^{a} A_{\mu}^{a}, t^{b} A_{\nu}^{b}\right]
$$

is the curvature tensor of the complex abstract vector space. Also we have used the matrix identity

$$
e^{A} e^{B}=e^{A+B+\frac{1}{2}[A, B]+\ldots}
$$

and the trace relations:

$$
\begin{array}{r}
\operatorname{tr}\left(t_{p}^{a}\right)=0 \\
\operatorname{tr}\left(t_{p}^{a} t_{p}^{b}\right)=C(p) \delta^{a b}
\end{array}
$$

where $C(p)$ is a constant for the representation $p$. As the system we are considering is subject to fluctuations and is not deterministic, the quantity which is actually of interest to us is the noise average $\left\langle\cos \theta_{y}-\cos \theta_{x}\right\rangle$ 
of the phase difference between $q_{1}$ and $q_{2}$ :

$\left\langle\cos \theta_{y}-\cos \theta_{x}\right\rangle=-\frac{C(p)}{2}\left\langle\int d^{d} x d^{d} y\left\{-i \Lambda^{a} F_{i j}^{a}+F_{i j}^{a} \int_{y}^{x} A_{\mu}^{a}(s) d s_{\mu}+\left(\delta^{\alpha a}+\frac{\epsilon^{\beta \alpha a}}{2} \Lambda^{\beta}\right) F_{i j}^{a} \int_{y}^{x} A_{\mu}^{\alpha}(s) d s_{\mu}+\ldots\right\}\right\rangle$

Using the Gauss-Bonnet theorem, we see that the first integral on the right hand side of this equation gives a topological invariant, the Euler characteristic $\chi_{E}$ of the surface $S$ over which the integration is performed: $\int_{S} F_{i j}=\chi_{E} . \quad$ For the situation in which there is perfect phase synchronization between any two oscillators in the system, this constant term on the right-hand side of eqn.(79) should vanish and the other terms in the equation must also vanish. The two-torus $T^{2}$ is a well known example of a topological space with vanishing Euler characteristic. The limit cycles of the coupled system are therefore constrained to remain on $T^{2}$ as they synchronize in phase.

For the oscillators $q_{1}$ and $q_{2}$ to exhibit phase-locked behaviour, we observe that we must have, to lowest order in $\Lambda$,

$$
\frac{C(p)}{2}\left\langle\int d^{d} x d^{d} y\left\{F_{i j}^{a}\left(2 \delta^{\alpha a}+\frac{\epsilon^{\beta \alpha a}}{2} \Lambda^{\beta}\right) \int_{y}^{x} A_{\mu}^{\alpha}(s) d s_{\mu}\right\}\right\rangle=\text { constant }
$$

While the explicit value of the left hand side of (80) would vary from one set of coupled systems to another, it is interesting to note that in all cases external noise seems to play a role in bringing about the phase synchronization. This can be seen as follows. The noise averages of $A_{\mu}$ and $F_{\mu \nu}$ are calculated by solving the coupled Langevin equations in (21) using eqns.(63) and (65). From eqns.(22),(48) and (63) we see that the lowest order terms of $\left\langle A_{\mu}\right\rangle$ and $\left\langle F_{\mu \nu}\right\rangle$ such as $\left\langle\left\langle\left\langle\pi_{f_{i}}\right\rangle\right\rangle\right\rangle$ would not contribute so that at least to this order, the left hand side of eqn.(80) is brought very close to zero taking the system towards synchrony, whereas in the case when no fluctuations are present these terms would be non-zero.

The role of noise in bringing about phase synchrony can also be understood in the following way. Consider the analysis by Ermentrout [27] of two weakly coupled oscillators:

$$
\frac{1}{\omega_{i}} \frac{d Z_{i}}{d t}=F_{i}\left(Z_{i}\right)+\kappa G_{i}\left(Z_{i}, Z_{j}\right), \quad i, j=1,2, \quad i \neq j
$$

$Z_{i} \in R^{N_{i}}, F_{i}$ are continuous and differentiable, $G_{i}$ are continuous, and each uncoupled system $\frac{d Z_{i}}{d t}=F_{i}\left(Z_{i}\right)$ admits a unique, globally stable periodic solution. It was shown in [27] that the coupled state admits a parameter regime in which $n: m$ phase-locking occurs between the two oscillators, after $n$ cycles of oscillator 1 and $m$ cycles of oscillator 2 . Using a multiple-scale perturbation technique, introducing slow $\tau$ and fast $s$ time variables: $\frac{d}{d t}=\omega_{2} \frac{d}{d s}+\kappa \frac{d}{d \tau}$, this $N_{1}+N_{2}$-dimensional system was reduced to a one-dimensional 
evolution equation on a slow time-scale for the phase difference $\Phi$ between the two oscillators. This was shown to have the form:

$$
\frac{d \Phi}{d \tau}=H(\Phi)
$$

where $H(\Phi)=H(\Phi+2 \pi)$, and the phase shifts $\Phi$ s vary slowly in the direction of the flow of the limit cycle which is formed due to the coupling of the oscillators. Further it was shown that the phase-locked solution to the coupled system corresponds to the fixed points of eqn.(82), $H$ being identified with the Poincare map for the flow of the full system.

For a nonzero value of the noise strength, the sharp transition to the critical point is replaced by a bifurcation region, and hence the time spent by the unstable modes near the bifurcation (critical slowing down of the deterministic system at the bifurcation) is much longer in the stochastic case.

It is known [28] that the slower the system moves along any part of the limit cycle, the larger is its statistical weight in that part of the limit cycle. Hence, from eqn.(82) and the result of [27] mentioned above, the phase-locked solutions of the coupled oscillators are statistically favoured. Moreover, since in the stochastic system these (unstable) slowly varying phase differences show an increase in the relaxation time as the instability is approached, as compared to the deterministic case, phase-locking and synchronous solutions have a larger statistical weight in the presence of the weak noise.

We have considered the case in which the coupled system exhibits limit cycle behaviour. The formation of a limit cycle involves symmetry-breaking, permitting the existence of both stationary and time-dependent probability densities (for finite and infinite system volumes respectively). The probability peaks for the time dependent densities rotate along the limit cycle, while the time-independent densities are crater shaped. A particular phase is associated with every given realization of a limit cycle and a choice made corresponds to breakdown of gauge symmetry. The emergent gauge structure associated with the phase degrees of freedom of the center modes in the vicinity of the bifurcation, enables us to introduce the geometrical quantities $A_{l}$ and $F_{l m}$, and to obtain the condition for phase-locking (eqn.(80)) in terms of these quantities.

Our analysis was made possible only because of the presence of weak noise which, as we showed in Section 3, plays the crucial part of enabling separability of the variables near the bifurcation into slow and fast ones evolving at different time scales.

Measurable phase differences between oscillators in the presence of the (weak) Gaussian white noise are 
noise-averaged quantities, and from eqn.(80), are determined largely by the fluctuations in the gauge potential. Since these average out to zero, we conclude that the presence of (weak) Gaussian white noise always enhances phase synchrony.

Recent experimental observations by Fujii et al [7] of two chemical oscillators separated by some distance in the light-sensitive Belousov-Zhabotinsky reaction show self-synchronization of phase and frequency by application of noise. They observed spontaneous synchronization for small separation distances in the absence of noise and demonstrated the existence of an optimum noise intensity for the self synchronization phenomenon. Phase synchronization in coupled non-identical FitzHugh-Nagumo neurons subject to independent external noise was also demonstrated through numerical simulations in [8]. Noise-induced phase and frequency synchronization was also demonstrated recently in stochastic oscillatory systems both analytically and with numerical simulations [9]. (see also [29]).

Our analysis provides a framework to understand these findings and opens avenues for deeper studies relating cooperative phenomena in coupled nonlinear stochastic systems, with the underlying rich geometrical structure of the phase space generated by the complex dynamics, and with associated mathematical invariants which govern the system's asymptotic behaviour.

\section{CONCLUSION}

We have introduced a geometrical approach aiming to understand phase synchronization among coupled nonlinear oscillators subject to additive noise. We have considered the specific scenario when the collective dynamics of all the oscillators also exhibits limit cycle behaviour arising via one or more Hopf bifurcations, consequently implying the occurrence of frequency synchronization. We demonstrate the emergence of a non Abelian gauge structure arising from the evolution of the slow dynamics but induced by the fast degrees of freedom. The condition required to be satisfied in order for phase locking and phase synchronization to be exhibited is obtained in terms of characteristic invariants of the surface generated by the dynamics of the system. We find that weak noise helps in bringing about phase synchronization. This provides an explanation of recent experimental observations and numerical simulations of noise-induced phase synchronization $[7,8]$ (see also $[9,29])$. Our work also motivates further studies of the internal structure and geometry of synchronization defects in spiral waves in oscillatory media which have been areas of keen interest in recent times $[30,31]$. 


\section{Acknowledgements}

I am grateful to V.Srinivasan, S.Chaturvedi, Wim van Saarloos, and Cornelis Storm for helpful discussions. I would like to acknowledge support from the Max Planck Institute for Mathematics in the Sciences, Leipzig

where most of this work was done, and warm hospitality of the Instituut-Lorentz, Universiteit Leiden where it was brought to completion.

\section{References}

1. A.Pikovsky, M.Rosenblum \& J.Kurths, Synchronization: A universal concept in nonlinear sciences, Cambridge University Press, Cambridge (2001).

2. M.G.Rosenblum, A.S.Pikovsky \& J.Kurths, Phys.Rev.Lett. 76, 1804 (1996);

G.V.Osipov, A.S.Pikovsky, M.G.Rosenblum \& J.Kurths, Phys.Rev.E 55, 2353 (1997);

S.K.Han, T.G.Yim, D.E.Postnov \& O.V.Sosnovtseva, Phys.Rev.Lett. 83, 1771 (1999).

3. H.Fujisaka \& T.Yamada, Prog.Theor.Phys. 69, 32 (1983);

A.S.Pikovsky, Z.Phys.B 55, 149 (1984);

L.M.Pecora \& T.L.Carroll, Phys.Rev.Lett. 64, 821 (1990).

4. A.Shapere \& F.Wilczek, Phys.Rev.Lett. 58, 2051 (1987).

5. A.Shapere \& F.Wilczek, J.Fluid.Mech. 198, 557 (1989).

6. A.Shapere \& F.Wilczek, in Geometric Phases in Physics, World Scientific Publishing Co, Singapore, (1989).

7. K.Fujii, D.Hayashi, O.Inomoto \& S.Kai, Forma 15, 219 (2000).

8. B.Hu \& C.Zhou, Phys.Rev.E 61, R1001 (2000).

9. J-n Teramae \& D.Tanaka, Phys.Rev.Lett. 93, 204103 (2004);

D.S.Goldobin \& A.Pikovsky, Phys.Rev.E 71, 045201(R) (2005).

10. E.Knobloch \& K.A.Weisenfeld, J.Stat.Phys 33, 611 (1983). 
11. C.Van den Broeck, M.Malek Mansour \& F.Baras, J.Stat.Phys. 28, 557 (1982);

F.Baras,M.Malek Mansour \& C.Van den Broeck, J.Stat.Phys. 28, 577 (1982).

12. H.Haken, Advanced synergetics : instability hierarchies of self-organizing systems and devices, SpringerVerlag, (1983).

13. E.Gozzi, Phys.Rev.D 28, 1922 (1983).

14. E.Gozzi \& W.D.Thacker, Phys.Rev.D 35, 2398 (1987).

15. V.I.Arnold, Mathematical methods of classical mechanics, (Second Edition), Springer-Verlag, (1989).

16. M.V.Berry, Proc.R.Soc.Lond.A 392, 45 (1984).

17. M.V.Berry, J.Phys.A 18, 15 (1985);

J.H.Hannay, J.Phys.A 18, 221 (1985);

M.V.Berry \& J.H.Hannay, J.Phys.A, 21, L325 (1988).

18. C.Lanczos, The variational principles of mechanics, Dover Publications, New York, (1986).

19. W.Magnus, Commun.Pure Appl.Math. 7, 649 (1954).

20. R.M.Wilcox, J.Math.Phys. 8, 962 (1967).

21. R.A.Marcus, J.Chem.Phys. 52, 4803 (1970).

22. M.Timme, F.Wolf \& T.Geisel, Phys.Rev.Lett. 89, 258701 (2002).

23. G.Giavarini, E.Gozzi, D.Rohrlich \& W.D.Thacker, Phys.Rev.D 39, 3007 (1989).

24. G.Giavarini \& E.Onofri, J.Math.Phys. 30, 659 (1989).

25. S.Chaturvedi, M.S.Sriram \& V.Srinivasan, J.Phys.A 20, L1071 (1987).

26. F.Wilczek \& A.Zee, Phys.Rev.Lett. 52, 2111 (1984).

27. G.B.Ermentrout, J.Math.Biol. 12, 327 (1981).

28. F.Baras, in Lecture notes in Physics -vol.484, ed. L.Schimansky-Geier \& T.Poeschel, pg.167, SpringerVerlag, (1997);

J.W.Turner, in Proceedings of the International onference on Synergetics, ed. H.Haken, p.255 (Springer, Berlin), (1980). 
29. L.Callenbach et al., Phys.Rev.E 65, 051110 (2002).

30. A.Goryachev, H.Chate \& R.Kapral, Phys.Rev.Lett. 80, 873 (1998); ibid., Int.J.Bif.Chaos, 10, 1537 (2000).

31. J.Davidsen \& R.Kapral, Phys.Rev.E 66, 055202 (2002). 